\title{
AIBP augments cholesterol efflux from alveolar macrophages to surfactant and reduces acute lung inflammation
}

\author{
Soo-Ho Choi, ${ }^{1}$ Aaron M. Wallace, ${ }^{2}$ Dina A. Schneider, ${ }^{1}$ Elianne Burg, ${ }^{2}$ Jungsu Kim, ${ }^{1}$ Elena Alekseeva, \\ Niki D.J. Ubags, ${ }^{2}$ Carlyne D. Cool, ${ }^{3}$ Longhou Fang, ${ }^{4,5}$ Benjamin T. Suratt, ${ }^{2}$ and Yury I. Miller ${ }^{1}$ \\ 'Department of Medicine, UCSD, La Jolla, California, USA. 'Department of Medicine, University of Vermont College of \\ Medicine, Burlington, Vermont, USA. ${ }^{3}$ Department of Pathology, University of Colorado Anschutz Medical Campus, \\ Aurora, Colorado, USA. ${ }^{4}$ Center for Cardiovascular Regeneration, Department of Cardiovascular Sciences, Houston \\ Methodist Research Institute, and ${ }^{5}$ Houston Methodist DeBakey Heart and Vascular Center, Houston Methodist, \\ Houston, Texas, USA.
}

Acute respiratory distress syndrome (ARDS) is characterized by an excessive pulmonary inflammatory response. Removal of excess cholesterol from the plasma membrane of inflammatory cells helps reduce their activation. The secreted apolipoprotein A-I binding protein (AIBP) has been shown to augment cholesterol efflux from endothelial cells to the plasma lipoprotein HDL. Here, we find that AIBP was expressed in inflammatory cells in the human lung and was secreted into the bronchoalveolar space in mice subjected to inhalation of LPS. AIBP bound surfactant protein $B$ and increased cholesterol efflux from alveolar macrophages to calfactant, a therapeutic surfactant formulation. In vitro, AIBP in the presence of surfactant reduced LPSinduced p65, ERK1/2 and p38 phosphorylation, and IL-6 secretion by alveolar macrophages. In vivo, inhalation of AIBP significantly reduced LPS-induced airspace neutrophilia, alveolar capillary leak, and secretion of IL-6. These results suggest that, similar to HDL in plasma, surfactant serves as a cholesterol acceptor in the lung. Furthermore, lung injury increases pulmonary AIBP expression, which likely serves to promote cholesterol efflux to surfactant and reduce inflammation.

Authorship note: BTS and YIM contributed equally to this work.

Conflict of interest: YIM and LF are inventors listed in patent applications related to the topic of this paper.

Submitted: February 13, 2018

Accepted: July 3, 2018

Published: August 23, 2018

Reference information: JCI Insight. 2018;3(16):e120519. https://doi.org/10.1172/jci. insight.120519.

\section{Introduction}

Receptors involved in inflammatory signaling, including TLR4, localize, constitutively or upon ligand binding, to lipid rafts, which are membrane microdomains characterized by high content of cholesterol and sphingomyelin (1-4). The decreased diffusion rates present in lipid rafts support TLR4 homodimerization, which is an obligatory step in its signaling cascade $(5,6)$. Cholesterol removal from the plasma membrane reduces lipid rafts and disrupts TLR4 signaling (7). Treatment with methyl- $\beta$-cyclodextrin $(\mathrm{M} \beta C D)$ is a common method to deplete cholesterol from the plasma membrane in cell culture experiments, which does result in inhibition of TLR4-mediated inflammatory responses $(8,9)$. However, the physiologic mechanism of cholesterol removal from plasma membrane involves ATP-binding cassette $(\mathrm{ABC})$ cholesterol transporters $\mathrm{ABCA} 1$ and ABCG1 and the extracellular cholesterol acceptors apoA-I and HDL (10-13). Consequently, there is a substantial increase in inflammatory gene expression in response to TLR ligands in $\mathrm{Abca1^{-/- }} \mathrm{Abcg} 1^{-/-}$cells (14, 15), and the cholesterol acceptors HDL and lipid-poor apoA-I reduce inflammatory responses (16-21).

We have reported that the secreted apoA-I binding protein (AIBP; gene name APOA1BP), which by itself does not bind cholesterol, facilitates cholesterol removal from endothelial cells to HDL (22). This results in a significantly greater reduction of lipid rafts in endothelial cells incubated with AIBP/HDL than with HDL alone; in disrupted cell-surface colocalization of caveolin-1 and VEGFR2, as well as decreased VEGFR2 and caveolin-1 localization to lipid rafts; in reduced VEGF-induced VEGFR2 dimerization; in VEGFR2 endocytosis; and in phosphorylation of VEGFR2, AKT, FAK, and SRC in endothelial cells stimulated with VEGF (22). These cell culture experiments were corroborated with experiments in embryonic zebrafish in which apoa1bp knockdown resulted in ectopic and excessive angiogenesis (22). Similar to the effect of AIBP on endothelial cells, AIBP has been reported to promote cholesterol efflux from THP-1derived and RAW macrophages and microglia (23-25). 
In this study, we tested the hypothesis that AIBP-mediated disruption of lipid rafts also affects activation of inflammatory receptors in alveolar macrophages. We focused on lung inflammation because the extracellular mechanism of AIBP action offers the possibility of its topical pulmonary delivery through inhalation. Function of TLRs, and particularly TLR4, in acute lung inflammation is well documented (26). TLR recognition of both exogenous pathogen-associated molecular patterns, such as LPS, and endogenous damage-associated molecular patterns, including fibronectin, hyaluronan, oxidized lipids, and heat shock proteins, drive activation of macrophages and neutrophils following lung infection or injury, which both initiate and sustain the inflammatory process. Furthermore, TLR4 signaling has recently been shown to be critical in the pathogenesis of ventilator-induced lung injury $(27,28)$.

This study also addressed the question of a pulmonary cholesterol acceptor. If AIBP-facilitated cholesterol removal from alveolar macrophages is indeed a relevant antiinflammatory mechanism in the lung, what is the cholesterol acceptor in the airspace? We tested the hypothesis that pulmonary surfactant serves as a cholesterol acceptor from alveolar macrophages and that AIBP augments surfactant-mediated efflux. Finally, this study examined the possibility of reducing acute lung injury in mice exposed to aerosolized AIBP.

\section{Results}

$A I B P$ is expressed in the inflamed lung. AIBP mRNA is ubiquitously expressed (29); the protein can be found in the nucleus, cytoplasm, and mitochondria and can also be secreted (22, 29, 30). However, AIBP expression under inflammatory conditions has heretofore not been investigated, to our knowledge. Immunostaining of human lung tissue revealed no detectable AIBP in the lung of subjects with no lung pathology, but AIBP-positive inflammatory cells were abundant in the lung of patients who died of bacterial pneumonia with acute respiratory distress syndrome (ARDS) (Figure 1A; representative images of 3 no lung pathology and 3 ARDS human specimens). In unchallenged mouse lung, AIBP is expressed in bronchial epithelium but not in alveolae (Figure 1B). However, inhaled LPS injury resulted, in 3 hours, in recruitment of inflammatory cells that express AIBP; at 6 hours after injury, AIBP was significantly increased in bronchoalveolar lavage fluid (BALF); tissue and BAL AIBP expression was observed up until 24 hours after exposure to inhaled LPS (Figure 1, B and C). Based on cell morphology, both neutrophils and macrophages express AIBP in injured lung (Figure 1, B and C, insets). These results suggest that in vivo AIBP is secreted into the airspace in response to injury and inflammation.

In vitro, stimulation of the MH-S alveolar macrophage cell line with LPS did not affect Apoalbp mRNA expression (Figure 2A). However, intracellular AIBP protein levels increased in response to LPS, reaching peak at 8 hours and returning to basal levels by 24 hours after stimulation (Figure 2B). Although no detectable AIBP was found in media, there was a significant increase in the cell surface-bound AIBP (Figure 2C). We interpret these data as demonstrating the binding of secreted AIBP back to the surface of activated macrophages, where it may serve to downregulate inflammatory response.

AIBP augments cholesterol efflux from alveolar macrophages to surfactant and reduces inflammatory responses. Pulmonary surfactant is a complex lipoprotein, consisting of surfactant protein A (SP-A), SP-B, SP-C, and SP-D; phospholipids; and cholesterol. Similar to apoA-I, which is a structural component necessary for HDL assembly, SP-B is a core SP, which has high affinity to phospholipids and is required for physical assembly of surfactant. Newborn $S f t p b^{-1-}$ mice die from respiratory failure (31). Thus, we tested whether AIBP binds SP-B. In a pull-down experiment, AIBP immunoprecipitated with Glutathione S-transferasetagged (GST-tagged) SP-B but to a much lesser degree with GST-tagged SP-D and not with GST-tagged c-FOS (irrelevant protein control) (Figure 3A).

Next, we tested the hypothesis that surfactant can serve as an acceptor in cholesterol efflux from alveolar macrophages. A clinically relevant surfactant preparation (INFASURF [calfactant] from ONY Laboratories) supported cholesterol efflux both from alveolar macrophage cell line MH-S and from primary murine alveolar macrophages. Remarkably, AIBP further significantly increased cholesterol efflux from alveolar macrophages to surfactant (Figure 3, B and C).

We hypothesized that the AIBP/surfactant-mediated cholesterol removal from alveolar macrophages would reduce ligand-mediated TLR4 activation in lipid rafts. It is well established that exposure to LPS rapidly inhibits cholesterol efflux and upregulated lipid rafts to gain support to inflammatory signaling $(32,33)$. Indeed, an increase in lipid rafts abundance was observed in $\mathrm{MH}-\mathrm{S}$ alveolar macrophages in response to LPS (Figure 3D). Pulmonary surfactant inhibited LPS-induced increases in lipid rafts, and AIBP further significantly augmented the surfactant's effect (Figure 3D). LPS-induced p65 and ERK1/2 
A
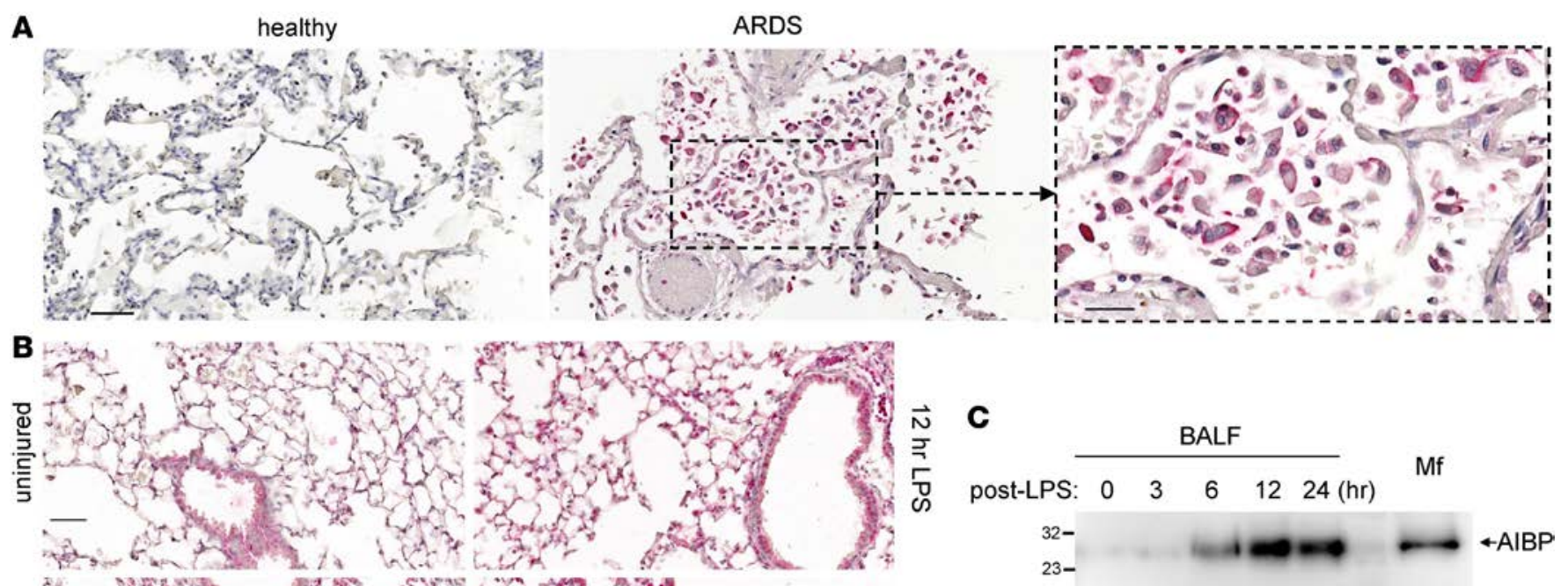

C
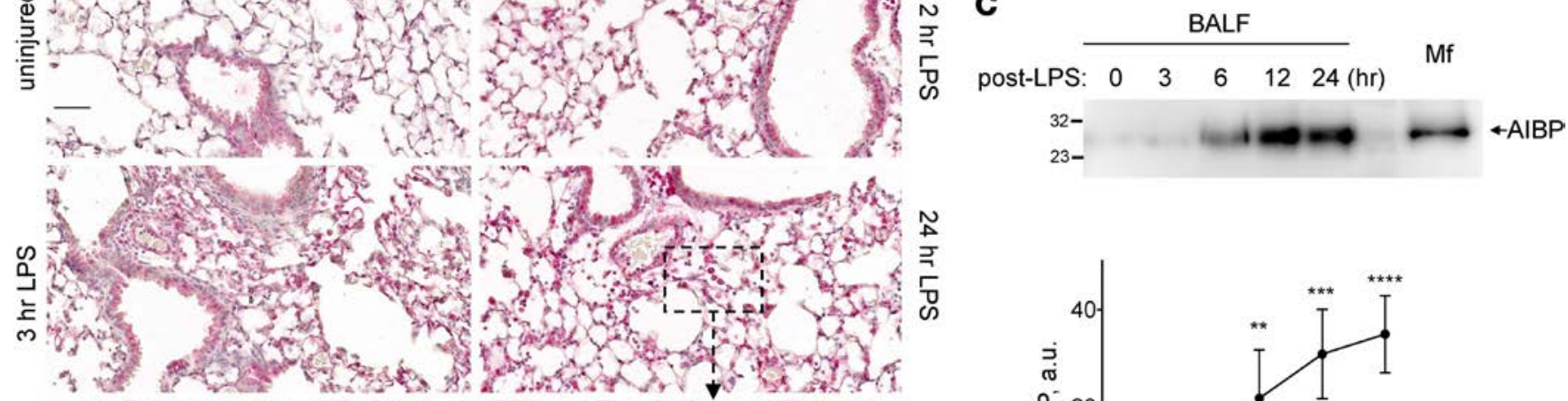

\section{$\frac{\Omega}{2}$}
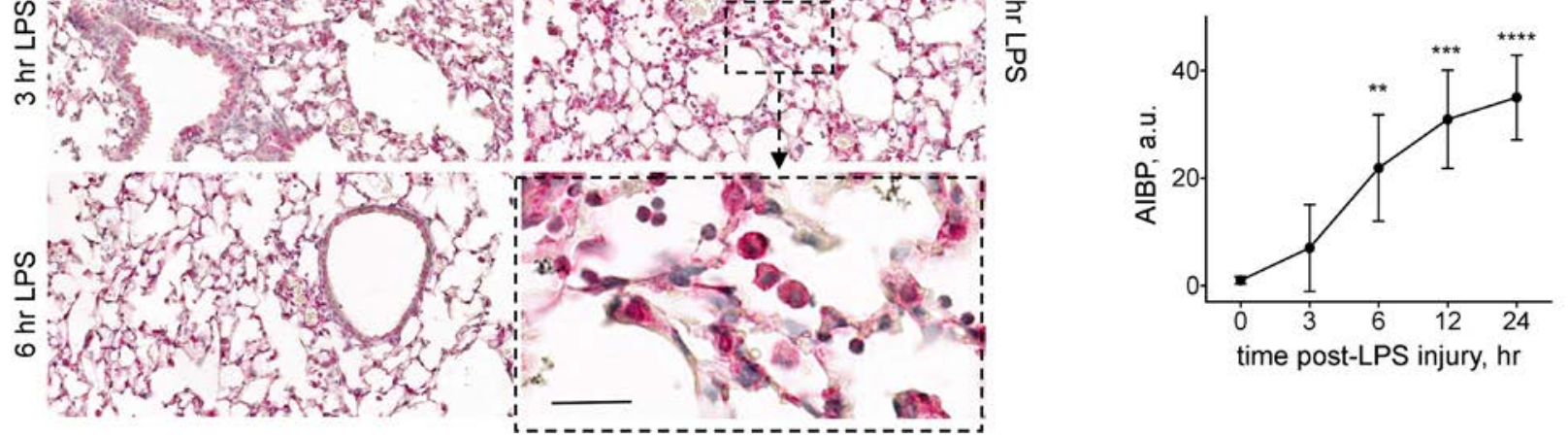

Figure 1. AIBP expression in inflamed lung. (A) Sections of lung tissues from a no lung pathology subject and a patient with ARDS (bacterial pneumonia) were stained with an anti-AIBP antibody (red) and counterstained with H\&E. The images are representative of sections from 3 no lung pathology and 3 ARDS subjects. (B) Mice were subjected to nebulized LPS, and BAL and lungs were collected at 3, 6, 12, and 24 hours after injury. Lung sections were stained with an anti-AIBP antibody (red) and counterstained with H\&E. (C) The BALF was run on SDS-PACE, and Western blots were probed with an antiAIBP antibody. The lysate of peritoneal macrophages, which express high levels of AIBP, was used as a positive control. Mean $\pm S E M(n=4) ;{ }^{* *} P<0.01$; ${ }^{* *} P<0.005 ;{ }^{* * *} P<0.001$ (1-way ANOVA with multiple comparisons). Scale bars: $50 \mu \mathrm{m}$ in $\mathbf{A}$ and $\mathbf{B}$, and $25 \mu \mathrm{m}$ in zoomed-in images (dashed boxes).

phosphorylation and IL-6 secretion to the media were inhibited in the presence of AIBP and surfactant, whereas surfactant alone had only minimal effect (Figure 4, A-D). Adding cholesterol back to the plasma membrane via incubation with cholesterol-M $\beta C D$ reversed the inhibitory effect of surfactant/ AIBP on p65, ERK1/2, and p38 phosphorylation in LPS-stimulated MH-S cells (Figure 5, A and B). AIBP by itself does not bind LPS (Supplemental Figure 1; supplemental material available online with this article; https://doi.org/10.1172/jci.insight.120519DS1) and is unlikely to reduce a pool of LPS available for TLR4 stimulation. These results suggest that the lung surfactant, aided by AIBP, serves to restrain inflammatory responses in alveolar macrophages.

Aerosolized AIBP reduces lung inflammation in mice. Because the inhalation of aerosolized LPS stimulated substantial AIBP secretion into the airspace (Figure 1C) and because AIBP reduced inflammatory signaling in alveolar macrophages (Figure 4 and Figure 5), we tested whether increasing AIBP levels in the airspace might reduce lung inflammation. First, we confirmed that nebulizing recombinant AIBP effectively delivers it to the airspace of the lung and found the recombinant AIBP in the BALF 1 hour after administration (Figure $6 \mathrm{~A}$ ). We estimated that approximately $60 \mathrm{ng}$ of recombinant AIBP was delivered to the lung using this approach. Next, we subjected mice to inhalation of aerosolized AIBP or BSA (control), followed 2 hours later by inhalation of aerosolized LPS. Twenty-four hours after LPS administration, mice were euthanized and the BAL was collected. Administration of AIBP resulted in significantly reduced airspace neutrophil recruitment, plasma albumin leakage, and IL-6 levels, as compared with the control mice receiving BSA (Figure 6, B-E). Nebulized BSA did not affect LPS-induced neutrophil recruitment (Supplemental Figure 2A). 
A

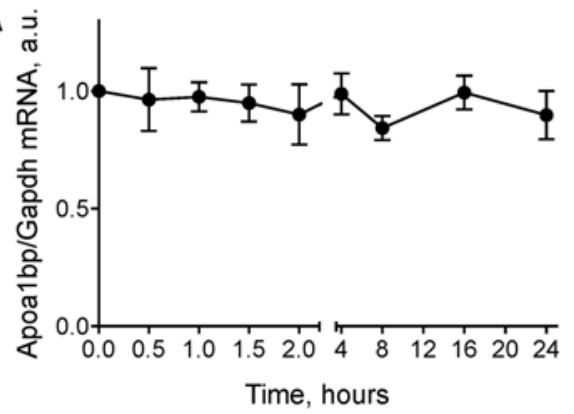

B

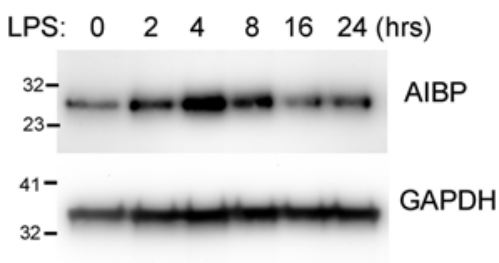

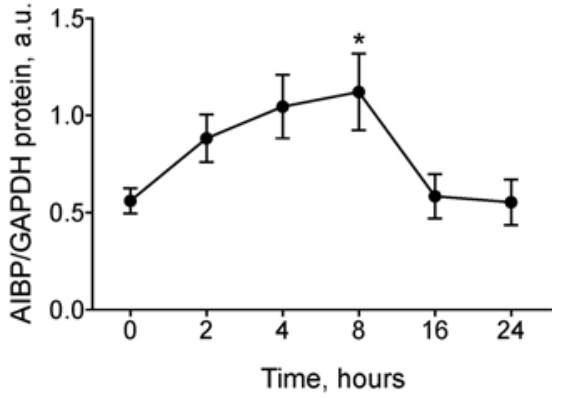
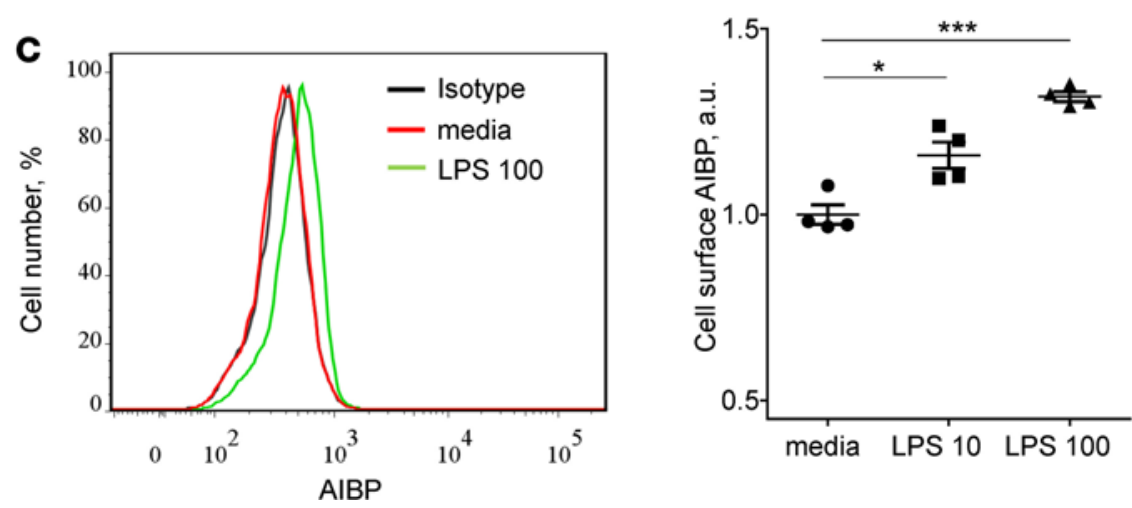

Figure 2. AIBP expression in activated MH-S alveolar macrophages. (A and B) MH-S cells were incubated with $10 \mathrm{ng} / \mathrm{ml}$ LPS for $0.5-24$ hours as indicated. (A) Apoa1bp mRNA expression. (B) Intracellular AIBP protein expression. (C) MH-S cells were incubated with 10 or $100 \mathrm{ng} / \mathrm{ml}$ LPS for 24 hours. Cells were not permeabilized and were stained with an anti-AIBP antibody. Cell surface AIBP expression was measured by flow cytometry. 7-AAD-positive cells were gated out. Mean $\pm \operatorname{SEM}(n=4) ;{ }^{*} P<0.05 ;{ }^{* *} P<0.001$ (1-way ANOVA with multiple comparisons).

\section{Discussion}

AIBP was discovered in a screen of proteins that physically associate with apoA-I (29). The human $A P O A 1 B P$ gene encoding the AIBP protein is located at 1q21.2-1q22 on chromosome 1. Human APOA1BP mRNA is ubiquitously expressed (29). Two studies of the AIBP protein structure have suggested it has an $\mathrm{NAD}(\mathrm{P}) \mathrm{H}$-hydrate epimerase or ADP-ribosyltransferase activity $(34,35)$, but so far, the physiological role of these enzymatic functions of AIBP has not been evaluated, to our knowledge. AIBP was implicated in oogenesis, spermiogenesis, and sperm capacitation, presumably through its involvement in cholesterol efflux $(36,37)$, but the latter was not experimentally addressed in vivo. Our work has demonstrated AIBP involvement in cholesterol efflux (22). Specifically in endothelial cells, AIBP binds both the cell surface and HDL, resulting in an increased number of binding sites for HDL and an increased dissociation constant, thereby creating conditions for faster HDL turnover and accelerated cholesterol efflux (22). In addition, as reported by Zhang et al. $(23,38)$, AIBP promotes apoA-I binding to ABCA1 at the cell surface of THP-1derived macrophages, prevents $\mathrm{ABCA} 1$ protein degradation, and thus reduces inflammatory response and foam cell formation. Overexpression of AIBP delivered by an adeno-associated virus reduces atherosclerosis in hypercholesterolemic mouse models $(24,39)$. In addition, spinal delivery of recombinant AIBP prevents neuropathic pain states in mice (25).

The mechanism by which AIBP augments cholesterol efflux requires its secretion from the cell, as replicated by adding recombinant AIBP to the cell culture media. Translated AIBP protein possesses a signal peptide $(29,36)$, although this has been disputed (30). AIBP secretion from kidney cells is induced by apoA-I (29) and regulated by PKA during sperm capacitation (36). We found that stimulation by LPS induces AIBP protein expression in MH-S alveolar macrophages and significantly increases AIBP levels detected on the cell surface. Because we previously reported that recombinant AIBP binds to endothelial cells (22), and to macrophages/microglia via TLR4 (25), we hypothesized that AIBP secreted by alveolar macrophages immediately binds back to the cell surface. This may constitute an autocrine or paracrine negative feedback mechanism in which AIBP that is secreted in response to inflammatory stimuli functions to downregulate inflammatory response. 
A
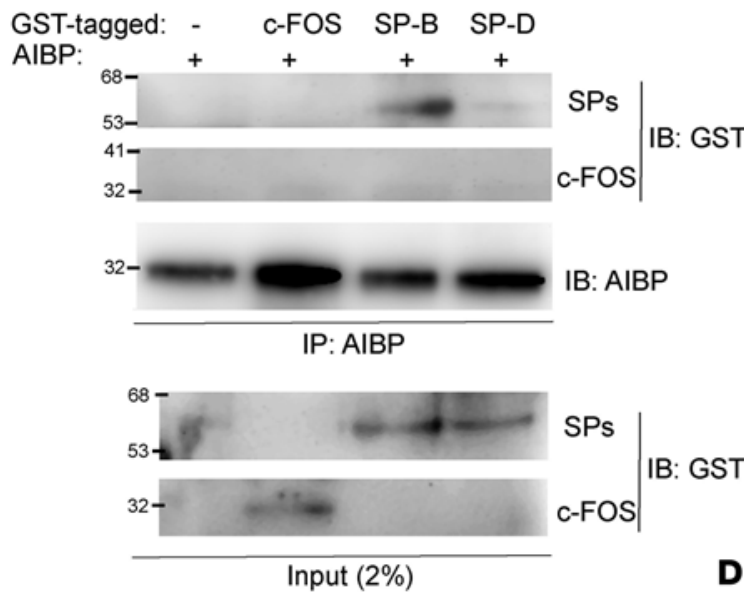

D

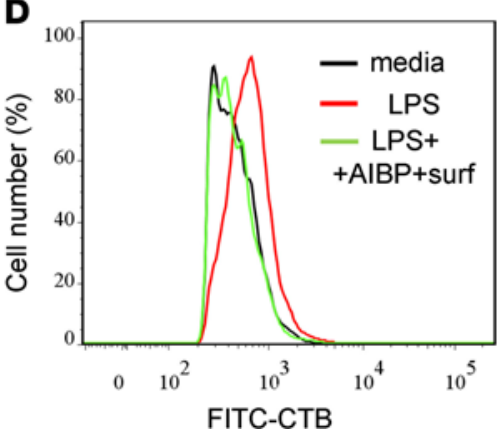

B

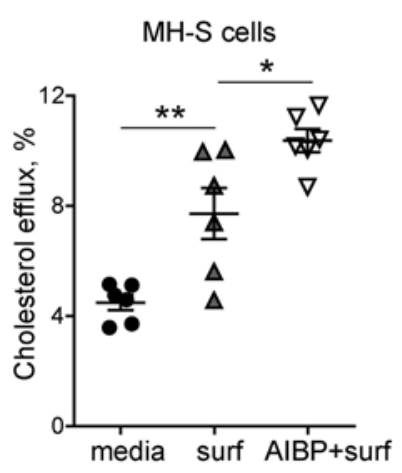

C

Primary alveolar

macrophages

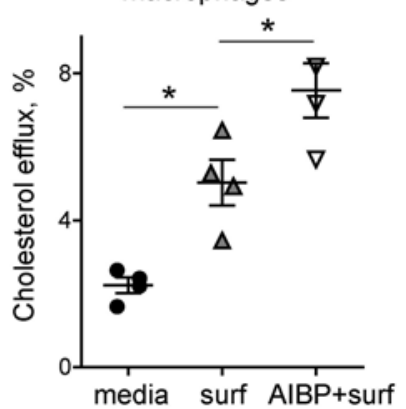

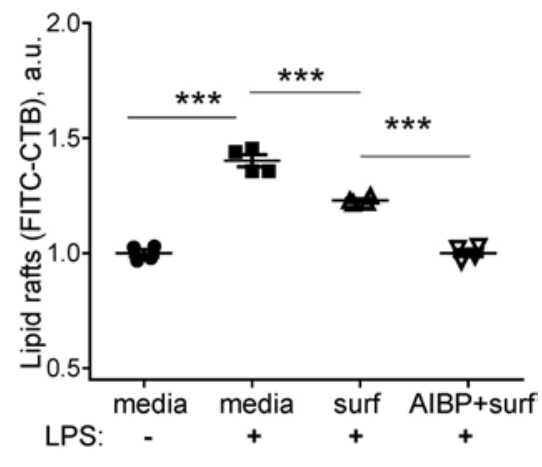

Figure 3. AIBP binds SP-B and augments surfactant-mediated cholesterol efflux. (A) GST-tagged c-FOS, SP-B, and SP-D (0.5 $\mu$ g) were incubated with $0.5 \mu \mathrm{g}$ of recombinant AIBP for 30 minutes at room temperature. AIBP was pulled downed with an anti-AlBP antibody. The blots of the pull-down and of input were probed with an anti-CST antibody. Representative blots from 3 independent experiments. (B and C) MH-S cells (B) and primary alveolar macrophages (C) were preloaded with ${ }^{3} \mathrm{H}$-cholesterol for 24 hours and further incubated with cAMP and an ACAT inhibitor for 18 hours. The cells were incubated for 2 hours with $50 \mu \mathrm{g} / \mathrm{ml}$ surfactant in the presence or absence of $0.2 \mu \mathrm{g} / \mathrm{ml} \mathrm{AIBP}$. The media and cell pellets were collected, and ${ }^{3} \mathrm{H}$ counts were measured. Efflux was calculated as the percentage of ${ }^{3} \mathrm{H}$ counts in the media divided by the sum of ${ }^{3} \mathrm{H}$ counts in the media and the cell pellet. (D) MH-S cells were incubated for 2 hours with $50 \mu \mathrm{g} / \mathrm{ml}$ surfactant in the presence or absence of $0.2 \mu \mathrm{g} / \mathrm{ml}$ AlBP, and then stimulated with $10 \mathrm{ng} / \mathrm{ml}$ LPS for 15 minutes. Content of CTB-positive lipid rafts was measured in a flow cytometry assay. Mean \pm SEM; $n=6(\mathbf{B})$ and $n=4(\mathbf{C}$ and $\mathbf{D})$; ${ }^{*} P<0.05 ;{ }^{*} P<0.01$; ${ }^{* *} P<0.0001$ (1-way ANOVA with multiple comparisons).

The present study not only extends the known cell types in which AIBP promotes cholesterol efflux to alveolar macrophages, but it also reports, for the first time to our knowledge, that AIBP can engage surfactant as the vehicle for cholesterol removal from alveolar macrophages. Further, we discovered that, specifically in the lung, administration of aerosolized AIBP reduces acute pulmonary inflammation in a mouse model relevant to human ARDS. Our findings demonstrate that not only does AIBP exposure lead to attenuated recruitment of the key cellular mediator of ARDS, neutrophils, but it also significantly reduces the occurrence of capillary leak, the hallmark finding of this disease. The mechanisms underlying these effects remain to be fully determined, but it is of note that AIBP also significantly reduced IL-6 release, which has been implicated in the pathogenesis of ARDS and has been shown to correlate with poor prognosis in the disease (40-42).

Our results suggest interesting parallels between an antiinflammatory function of HDL in plasma and the function of pulmonary surfactant in the airspace. Secretion of surfactant into the airspace makes the lung the third-most active lipid-secreting organ after the liver and intestine (43). Similar to HDL, surfactant is a complex lipoprotein, consisting of phospholipids, cholesterol, and proteins, and it is normally recycled from the airspace back into alveolar macrophages and type II cells. Importantly, the cholesterol transporter ABCG1, which mediates cholesterol efflux to HDL and is required for the AIBP effect in endothelial cells (22), is highly expressed in alveolar macrophages and type II cells, and the loss of ABCG1 results in excessive accumulation of lipids in the lung and in a major imbalance in surfactant lipid levels (43).

Surfactant dysfunction and loss are critical contributors to the pathogenesis of ARDS. Interestingly, although surfactant replacement has been shown to be very effective in the treatment of neonatal respiratory distress syndrome, a disease resembling ARDS that is caused by surfactant production failure in 
A

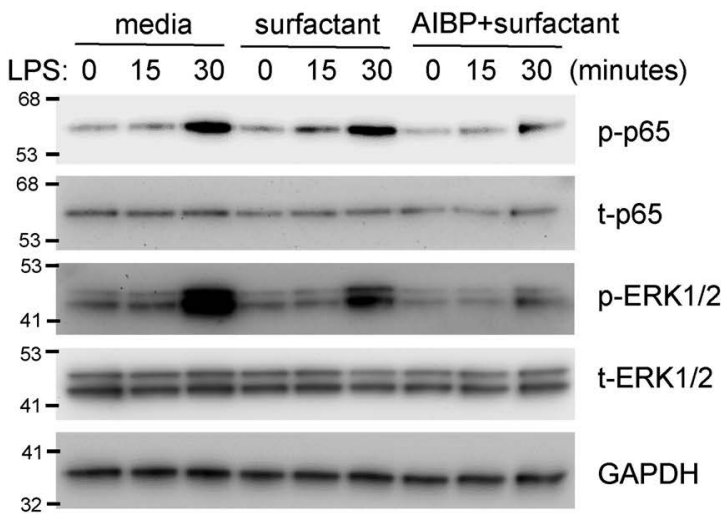

B

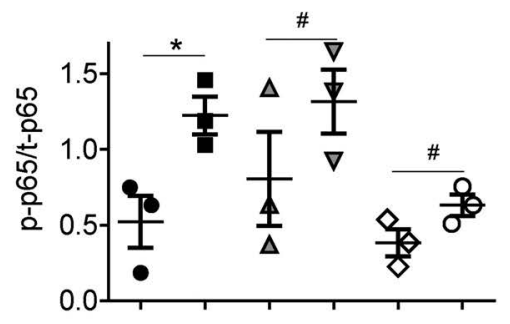

C
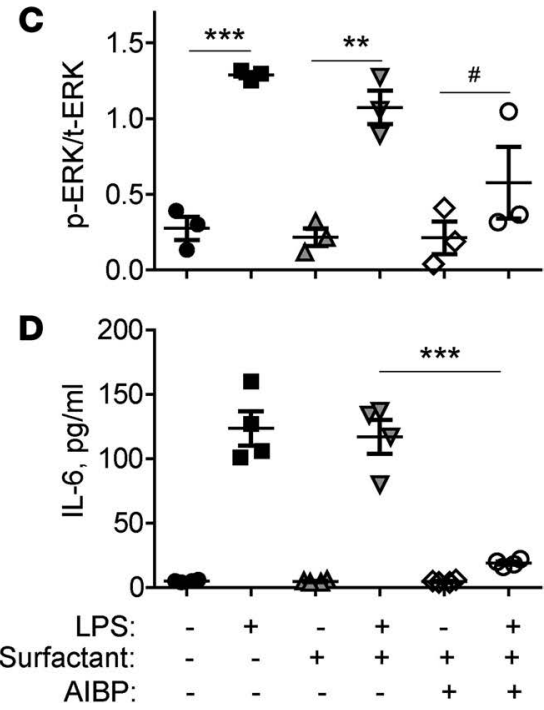

Figure 4. AIBP inhibits LPS-induced inflammatory responses in alveolar macrophages. MH-S cells were preincubated for 2 hours with $50 \mu \mathrm{g} / \mathrm{ml}$ surfactant in the presence or absence of $0.2 \mu \mathrm{g} / \mathrm{ml}$ AIBP and then stimulated with $10 \mathrm{ng} /$ mI LPS for the indicated times. (A) Representative immunoblot. (B and C) Quantitative data for phospho-p65 and phospho-ERK1/2 (30-minute time point). (D) MH-S cells were incubated under the same conditions for 6 hours, and the media were analyzed for IL-6 levels by ELISA. Mean \pm SEM; $n=3$ (B and C) and $n=4$ (D); ${ }^{*} P<0.05 ;{ }^{* *} P<0.01$; ${ }^{* * *} P<$ 0.001 ; \#, nonsignificant differences between 0 and 30-minute time points (2-tailed Student's $t$ test).

premature infants (44), such therapy has not been effective in adults with ARDS $(45,46)$, raising the possibility that additional critical airspace mediators may be deficient or dysfunctional in ARDS (47). Based on these findings and the noted similarities between surfactant and HDL, we propose that surfactant can serve as a cholesterol acceptor, that AIBP uses surfactant to regulate lipid rafts and inflammatory responses in alveolar macrophages, and that such interactions may be critical to attenuate the progression of ARDS.

\section{Methods}

IHC of lung tissue. Banked human lung specimens were analyzed from patients $(n=3)$ who died of bacterial pneumonia with ARDS and compared with histologically normal lung tissue $(n=3)$. Paraffin-embedded sections were deparaffinized, and antigens were demasked by incubation in a sodium citrate buffer for 30 minutes at $95^{\circ} \mathrm{C}$. Slides were stained with a rabbit anti-human AIBP antibody (Novus, NBP1-55934), followed by a biotinylated goat anti-rabbit IgG antibody (Vector Laboratories, BA-1000), an alkaline phosphatase-conjugated (AP-conjugaged) avidin/biotinylated enzyme complex (Vector Laboratories, AK-5000), and vector red substrate (Vector Laboratories, SK-5100). The same protocol was used for paraffin-embedded mouse tissue.

Cells. The transformed murine alveolar macrophage cell line MH-S was purchased from ATCC (CRL2019). The MH-S cells were maintained in RPMI-1640 (Corning Cellgro) supplemented with 10\% FBS (Omega Scientific), $0.05 \mathrm{mM}$ 2-mercaptoethanol, and $50 \mu \mathrm{g} / \mathrm{ml}$ gentamicin (all from Omega Scientific) Primary alveolar macrophages were isolated from the BAL of unchallenged mice. The BALF was collected by lavaging the lung 3 times with $0.8 \mathrm{ml}$ PBS containing $0.1 \%$ BSA via a tracheal catheter as previously described (48). BAL was spun down at $400 \mathrm{~g}$ for 5 minutes at room temperature. The cells were resuspended in RPMI-1640 supplemented with 5\% FBS and $50 \mu \mathrm{g} / \mathrm{ml}$ gentamicin.

Recombinant AIBP. For in vitro experiments, AIBP was produced in a baculovirus/insect cell system to ensure endotoxin-free preparation. Human AIBP was cloned into a pAcHLT-C vector (BD Biosciences) 
A

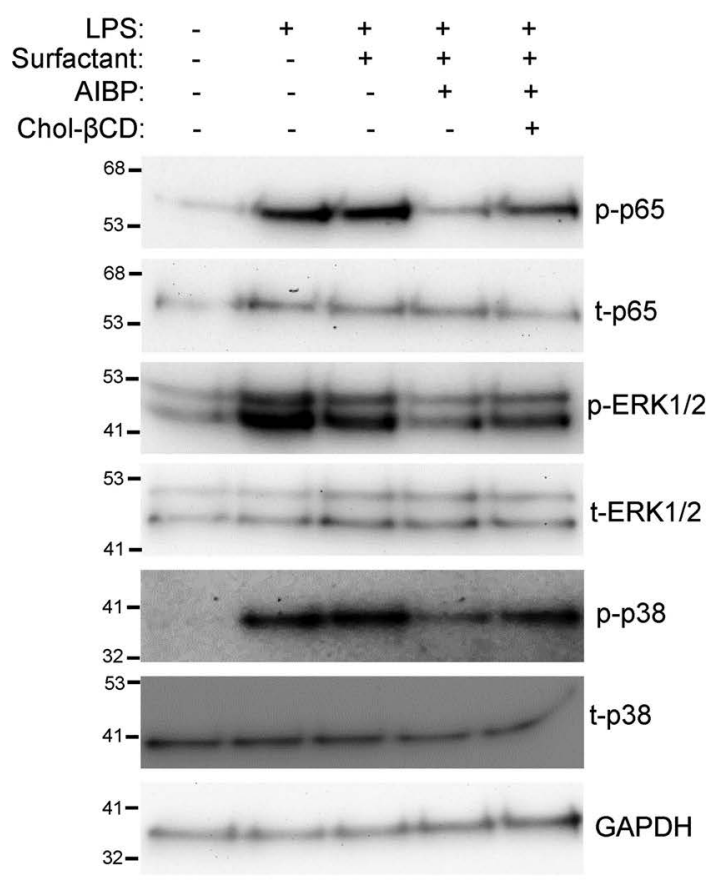

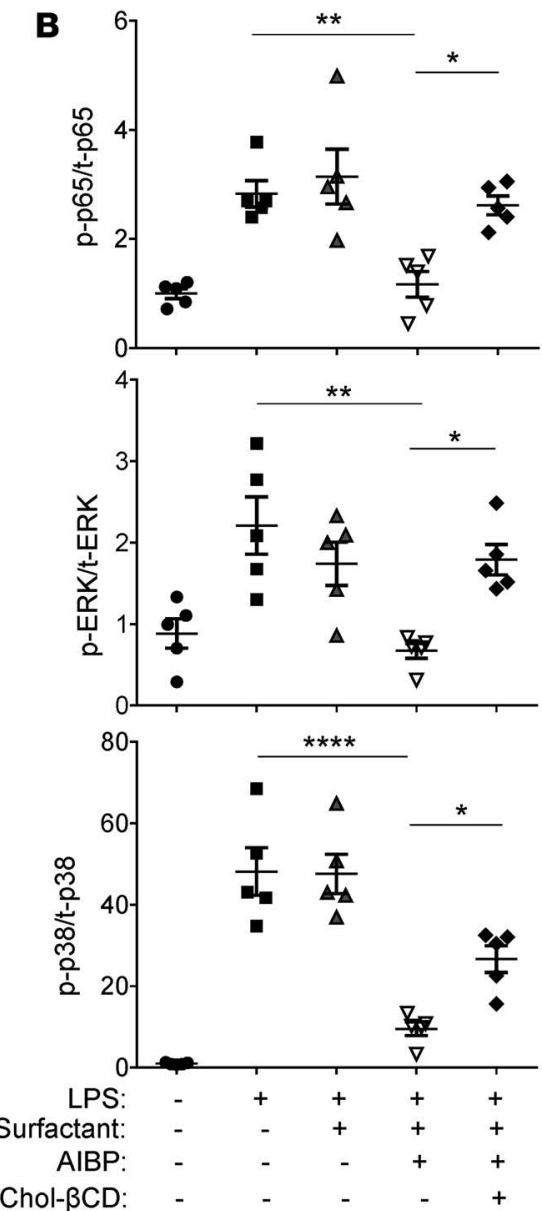

Figure 5. Adding cholesterol reverses antiinflammatory effect of AIBP. MH-S cells were preincubated for 2 hours with $50 \mu \mathrm{g} / \mathrm{ml}$ surfactant in the presence or absence of $0.2 \mu \mathrm{g} / \mathrm{ml}$ AIBP, followed by a 1-hour treatment with or without 50 $\mu \mathrm{g} / \mathrm{ml}$ cholesterol methyl- $\beta$-cyclodextrin (chol- $\beta C D$ ). The cells were then stimulated with $10 \mathrm{ng} / \mathrm{ml}$ LPS for 30 minutes. (A) Representative immunoblot. (B) Quantitative data for phospho-p65, phospho-ERK1/2, and phospho-p38. Mean \pm SEM; $n=5 ;{ }^{*} P<0.05 ;{ }^{* *} P<0.01 ;{ }^{* * *} P<0.0001$ (1-way ANOVA with multiple comparisons).

behind the polyhedrin promoter. The vector contains an N-terminal His-tag to enable purification and detection. Insect Sf9 cells (Thermo Fisher Scientific) were transfected with BD BaculoGold Baculovirus DNA (BD Biosciences) and the AIBP vector. After 4-5 days, the supernatant was collected to afford a baculovirus stock. Fresh Sf9 cells were infected with the AIBP producing baculovirus; cell pellets were collected after 3 days, which were lysed, sonicated, and cleared by centrifugation; and the supernatants were loaded onto a Ni-NTA agarose column (Qiagen) eluted with imidazole. Protein was dialyzed against PBS, and concentration was measured. Aliquots were stored at $-80^{\circ} \mathrm{C}$. To produce the bulk of AIBP for mouse lung injury experiments, AIBP was produced in E.coli as previously described (22).

AIBP-surfactant pull-down assay. GST-tagged, full-length human recombinant SP-B and SP-D were purchased from Abnova (H00006439-P01 and H00006441-P01, respectively). Recombinant AIBP (purified from an insect cells/baculovirus system) was incubated for 30 minutes with either SP-B, SP-D, or a GSTc-Fos fragment (300-380 aa) (49) in buffer ( $50 \mathrm{mM}$ Tris-HCl, pH 7.5, 1\% NP-40, $150 \mathrm{mM} \mathrm{NaCl}, 1 \mathrm{mM}$ EDTA, $1 \mathrm{mM}$ EGTA, $5 \mathrm{mM} \mathrm{Na}_{3} \mathrm{VO}_{4}, 1 \mathrm{mM} \mathrm{NaF}$, and protease inhibitor cocktail from MilliporeSigma) at room temperature. Protein A/G sepharose beads (Genesee Scientific, 20-528 and 20-537) were added for another 30-minute incubation at $4^{\circ} \mathrm{C}$ and immunoprecipitated with a rabbit anti-AIBP antibody (Abcam, ab75114) overnight at $4^{\circ} \mathrm{C}$. The next day, the samples were further incubated with protein $A / G$ sepharose beads for additional 1 hour at $4^{\circ} \mathrm{C}$. Immune complexes were washed 5 times with the buffer and run on a NuPAGE gel (Invitrogen), and AIBP-bound SPs was detected by immunoblotting with a mouse anti-GST antibody (Thermo Fisher Scientific, 8-326). 
A

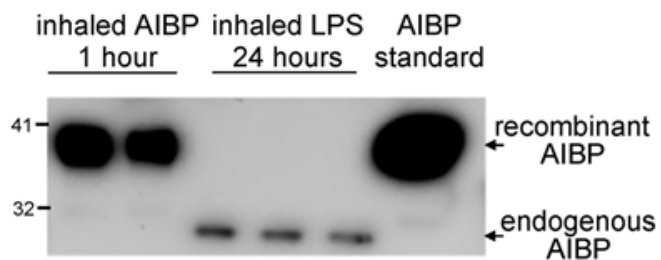
AlBP

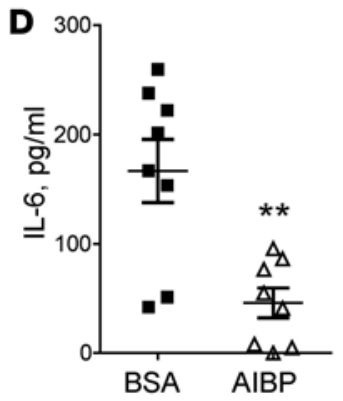

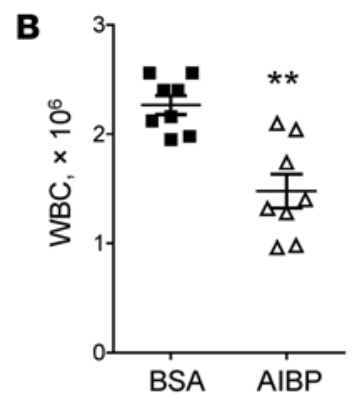

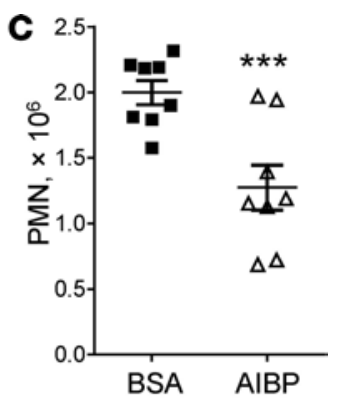

Figure 6. Inhalation of AIBP reduces lung injury. (A) Two mice were nebulized with AIBP, and 3 mice were nebulized with LPS. The BAL was obtained 1 hour or 24 hours later, respectively. BALF were subjected to SDS-PAGE and immunoblot with an anti-AIBP antibody. (B-E) Mice were nebulized with AIBP and, 2 hours later, nebulized with LPS. The BAL was obtained 24 hours after administration of LPS and analyzed for white blood cell (B) and polymorphonuclear cell (C) counts, IL-6 (D), and mouse albumin (E). Mice nebulized with BSA instead of AIBP were used as a reference group. Mean $\pm \mathrm{SEM} ; n=8 ;{ }^{*} P<$ $0.05 ;{ }^{*} P<0.01 ;{ }^{* *} P<0.001$ (2-tailed Student's $t$ test).

Cholesterol efflux assay. The cholesterol efflux assay was performed as described in the previous study (22), with modifications. In brief, MH-S cells or primary alveolar macrophages were loaded with $2 \mu \mathrm{Ci} / \mathrm{ml}$ ${ }^{3} \mathrm{H}$-cholesterol (PerkinElmer) in RPMI-1640 supplemented with 10\% FBS. Twenty-four hours later, cells were washed with equilibration medium (RPMI- 1640 containing $0.2 \%$ BSA) and incubated with equilibration medium supplemented with $0.3 \mathrm{mM}$ cAMP (MilliporeSigma, C6885) and $3 \mu \mathrm{g} / \mathrm{ml}$ an acetyl-coA acetyltransferase (ACAT) inhibitor (MilliporeSigma, S9318) for 18 hours. Cells were washed with equilibration medium, and cholesterol efflux was initiated by addition of equilibration medium with $50 \mu \mathrm{g} / \mathrm{m}$. surfactant (INFASURF [calfactant] from ONY Laboratories), in the presence or absence of $0.2 \mu \mathrm{g} / \mathrm{ml}$ AIBP. After 2 hours of incubation, the medium was collected, and ${ }^{3} \mathrm{H}$ counts were measured with a multipurpose liquid scintillation counter LS 6500 (Beckman Coulter). Cells were extracted with 2-propanol, and the lipid extract was evaporated under argon, resuspended in 2-propanol, added to ScintiVerse BD Cocktail (Thermo Fisher Scientific), and counted. Cholesterol efflux was expressed as a percentage of ${ }^{3} \mathrm{H}$ counts in the medium compared with combined ${ }^{3} \mathrm{H}$ counts in the cells and the medium.

Immunoblot. MH-S cells were plated at $0.5 \times 10^{6}$ cells/well in a 12-well plate. The next day, cells were pretreated with serum-free RPMI-1640 supplemented with surfactant in the presence or absence of $0.2 \mu \mathrm{g} / \mathrm{ml}$ AIBP. After a 2-hour incubation, cells were stimulated with DMSO (vehicle) or LPS for the indicated time. In some experiments, cells were incubated for 1 hour with or without $50 \mu \mathrm{g} / \mathrm{ml}$ cholesterol-M $\beta C D$ (MilliporeSigma, C4951). Cells were harvested and subjected to a 4-12\% Nu-PAGE gel (Invitrogen) electrophoresis and immunoblotted with antibodies against phospho-p65, p65, phospho-ERK1/2, ERK1/2, phospho-p38, p38, and GAPDH (Cell Signaling Technology; 3033, 4764, 9103, 4695, 8690, 4511, and 2119, respectively) (49). For BALF, samples were separated on a 4-12\% Nu-PAGE gel and immunoblotted with antibodies against AIBP (guinea pig polyclonal as reported; ref. 22) or murine albumin (Cell Signaling Technology, 4929). The murine albumin antibody did not cross-react with BSA (Supplemental Figure 2B).

ELISA. Levels of IL-6 were measured using a mouse IL-6 DuoSet ELISA (R\&D Systems) according to the manufacturer's instructions.

Quantitative PCR. Quantitative PCR (qPCR) was performed as previously described $(24,25)$. Total RNA was isolated using Nucleospin RNA columns (Clontech). Isolated RNA was reverse transcribed using 
RNA to cDNA EcoDry (Clontech) following the manufacturer's protocol. qPCR was performed using KAPA SYBR FAST Universal qPCR kit (KAPA Biosystems), with the primers ordered from Integrated DNA Technologies (IDT) and a Rotor Gene Q thermocycler (Qiagen).

Flow cytometry assay for surface AIBP. MH-S cells were incubated with DMSO (vehicle) or LPS for 24 hours. Cells were fixed with $3.7 \%$ formaldehyde for 30 minutes and blocked with $2 \%$ normal mouse serum containing an anti-CD16/CD32 antibody (FcR $\gamma$ blocker, BD Bioscience, catalog 553141) for 30 minutes on ice. Cells were incubated with $1 \mu \mathrm{g} / \mathrm{ml}$ anti-AIBP antibody (Abcam, ab75114) for 1 hour at $4^{\circ} \mathrm{C}$, washed 2 times with PBS, and incubated with $1 \mu \mathrm{g} / \mathrm{ml}$ goat anti-rabbit Alexa Fluor 488 (Invitrogen, A-11034) for 1 hour at $4^{\circ} \mathrm{C}$. After 5 washes with PBS, cells were incubated with 7-AAD (BD Bioscience, 559925) for 15 minutes at room temperature. 7-AAD-negative cells were analyzed using a FACSCanto II (BD Biosciences) flow cytometer. Geometric means of FACS histograms were measured and presented as bar graphs.

Flow cytometry analysis of lipid rafts. Flow cytometry analysis of lipid rafts was performed as previously described $(24,25)$. In brief, MH-S cells were washed 2 times with a FACS buffer, fixed with $3.7 \%$ formaldehyde for 15 minutes on ice, washed 3 times with a FACS buffer, incubated with FITC-conjugated cholera toxin B (CTB; MillipoerSigma, C1665) for 1 hour on ice, and analyzed using a FACSCanto II (BD Biosciences) flow cytometer. Geometric means of FACS histograms were measured and presented as bar graphs.

LPS binding assay. A 96-well plate was coated with LPS $(1 \mu \mathrm{g} / \mathrm{ml}$; ultra-pure LPS-EB from InvivoGen, tlrl-3pelps) in PBS-EDTA containing $0.05 \%$ Triton X-100 and incubated overnight at room temperature (50). The next day, the plate was washed 3 times with PBS containing $0.05 \%$ Tween- 20 and blocked by adding PBS containing 1\% BSA for 1 hour at room temperature. Serially diluted of BSA, MD2 (R\&D Systems), or AIBP were added, and the plates were incubated for 3 hours at room temperature. For competition, $20 \mu \mathrm{g} / \mathrm{ml}$ of LPS was mixed with each protein and preincubated for 10 minutes at room temperature before being added to wells. A mouse anti-His monoclonal antibody (Thermo Fisher Scientific, MA121315; $2 \mu \mathrm{g} / \mathrm{ml}$ ) was incubated for 1 hour at room temperature. AP-conjugated anti-mouse IgG (Thermo Fisher Scientific, PA1-28755) was added and incubated for 1 hour at room temperature. Lumiphos 530 (Lumigen) was added as an AP substrate for 90 minutes, and luminescence was measured using a Dynex luminometer (Dynex Technologies). Data are expressed as relative light units counted per 100 milliseconds.

Mouse acute lung injury model. Murine LPS-induced lung injury was performed as previously described (51). Briefly, Mice were exposed to aerosolized E. coli 0111:B4 LPS (MilliporeSigma) using a $3 \mathrm{mg} / \mathrm{ml}$ solution of LPS in sterile saline nebulized by a Pari LC Plus Reusable Nebulizer with ProNeb Turbo Air Compressor (Pari Respiratory Equipment). The nebulizer was connected, via a central manifold, to a multicompartment pie-shaped Plexiglas aerosol chamber (modified Tepper box; ref. 52) in which groups of mice were exposed individually but simultaneously to the aerosol for 15 minutes. The same procedure was used to expose mice to nebulized $0.4 \mathrm{mg} / \mathrm{ml}$ AIBP or BSA in sterile saline.

Statistics. Results were analyzed using Student's $t$ test (2-tailed, for differences between 2 groups) or 1-way ANOVA (for multiple groups), using GraphPad Prism. A $P$ value less than 0.05 was considered significant.

Study approval. All patient specimens were examined in accordance with an approved IRB protocol (COMIRB Protocol 14-0329). Animal experiments were approved by the IACUC of the UCSD (protocol S04155) and the University of Vermont.

\section{Author contributions}

Conception and design contributed by YIM, BTS, LF, and SHC. Material collection and experiments contributed by SHC, AMW, DAS, EB, JK, EA, NDJU, CDC, and LF. Drafting of the manuscript contributed by YIM, BTS.

\section{Acknowledgments}

This study was supported by grants HL135737, HL136275, HL088093 (YIM), HL114734, and HL132155 (LF) from the NIH and grant SDG14710028 (SHC) from the American Heart Association. The UCSD Microscopy Core is supported by P30NS047101 from the NIH.

Address correspondence to: Yury Miller, Department of Medicine, University of California, San Diego, 9500 Gilman Drive, La Jolla, California 92093, USA. Phone: 858.822.5771; Email: yumiller@ucsd.edu. 
1. White CR, Smythies LE, Crossman DK, Palgunachari MN, Anantharamaiah GM, Datta G. Regulation of pattern recognition receptors by the apolipoprotein A-I mimetic peptide 4F. Arterioscler Thromb Vasc Biol. 2012;32(11):2631-2639.

2. Villacorta L, et al. Electrophilic nitro-fatty acids inhibit vascular inflammation by disrupting LPS-dependent TLR4 signalling in lipid rafts. Cardiovasc Res. 2013;98(1):116-124.

3. D'Alessio A, Kluger MS, Li JH, Al-Lamki R, Bradley JR, Pober JS. Targeting of tumor necrosis factor receptor 1 to low density plasma membrane domains in human endothelial cells. J Biol Chem. 2010;285(31):23868-23879.

4. Levitan I, Shentu TP. Impact of oxLDL on Cholesterol-Rich Membrane Rafts. J Lipids. 2011;2011:730209.

5. Schmitz G, Orsó E. CD14 signalling in lipid rafts: new ligands and co-receptors. Curr Opin Lipidol. 2002;13(5):513-521.

6. Triantafilou M, Triantafilou K. Membrane partitioning: is location everything when it comes to endotoxin recognition? Subcell Biochem. 2010;53:173-184.

7. Fessler MB, Parks JS. Intracellular lipid flux and membrane microdomains as organizing principles in inflammatory cell signaling. J Immunol. 2011;187(4):1529-1535.

8. Meng G, Liu Y, Lou C, Yang H. Emodin suppresses lipopolysaccharide-induced pro-inflammatory responses and NF-кB activation by disrupting lipid rafts in CD14-negative endothelial cells. Br J Pharmacol. 2010;161(7):1628-1644.

9. Shridas P, Bailey WM, Talbott KR, Oslund RC, Gelb MH, Webb NR. Group X secretory phospholipase A2 enhances TLR4 signaling in macrophages. J Immunol. 2011;187(1):482-489.

10. Bodzioch M, et al. The gene encoding ATP-binding cassette transporter 1 is mutated in Tangier disease. Nat Genet. 1999;22(4):347-351

11. Rust S, et al. Tangier disease is caused by mutations in the gene encoding ATP-binding cassette transporter 1 . Nat Genet. 1999;22(4):352-355.

12. Klucken J, et al. ABCG1 (ABC8), the human homolog of the Drosophila white gene, is a regulator of macrophage cholesterol and phospholipid transport. Proc Natl Acad Sci USA. 2000;97(2):817-822.

13. Tall AR, Yvan-Charvet L. Cholesterol, inflammation and innate immunity. Nat Rev Immunol. 2015;15(2):104-116.

14. Yvan-Charvet L, et al. Increased inflammatory gene expression in ABC transporter-deficient macrophages: free cholesterol accumulation, increased signaling via toll-like receptors, and neutrophil infiltration of atherosclerotic lesions. Circulation. 2008;118(18):1837-1847.

15. Sun Y, et al. Free cholesterol accumulation in macrophage membranes activates Toll-like receptors and p38 mitogen-activated protein kinase and induces cathepsin K. Circ Res. 2009;104(4):455-465.

16. Mineo C, Shaul PW. Regulation of signal transduction by HDL. J Lipid Res. 2013;54(9):2315-2324.

17. Terasaka N, et al. ABCG1 and HDL protect against endothelial dysfunction in mice fed a high-cholesterol diet. J Clin Invest. 2008;118(11):3701-3713.

18. van der Westhuyzen DR, de Beer FC, Webb NR. HDL cholesterol transport during inflammation. Curr Opin Lipidol. 2007;18(2):147-151.

19. Murphy AJ, et al. High-density lipoprotein reduces the human monocyte inflammatory response. Arterioscler Thromb Vasc Biol. 2008;28(11):2071-2077.

20. Muller C, Salvayre R, Nègre-Salvayre A, Vindis C. HDLs inhibit endoplasmic reticulum stress and autophagic response induced by oxidized LDLs. Cell Death Differ. 2011;18(5):817-828.

21. Zheng C, Aikawa M. High-density lipoproteins: from function to therapy. J Am Coll Cardiol. 2012;60(23):2380-2383.

22. Fang L, et al. Control of angiogenesis by AIBP-mediated cholesterol efflux. Nature. 2013;498(7452):118-122.

23. Zhang M, et al. Apolipoprotein A-1 binding protein promotes macrophage cholesterol efflux by facilitating apolipoprotein A-1 binding to ABCA1 and preventing ABCA1 degradation. Atherosclerosis. 2016;248:149-159.

24. Schneider DA, et al. AIBP protects against metabolic abnormalities and atherosclerosis. J Lipid Res. 2018;59(5):854-863

25. Woller SA, et al. Inhibition of Neuroinflammation by AIBP: Spinal Effects upon Facilitated Pain States. Cell Rep. 2018;23(9):2667-2677

26. Tolle LB, Standiford TJ. Danger-associated molecular patterns (DAMPs) in acute lung injury. J Pathol. 2013;229(2):145-156

27. Jiang D, et al. Regulation of lung injury and repair by Toll-like receptors and hyaluronan. Nat Med. 2005;11(11):1173-1179.

28. Vaneker M, et al. Low-tidal-volume mechanical ventilation induces a toll-like receptor 4-dependent inflammatory response in healthy mice. Anesthesiology. 2008;109(3):465-472.

29. Ritter M, et al. Cloning and characterization of a novel apolipoprotein A-I binding protein, AI-BP, secreted by cells of the kidney proximal tubules in response to HDL or ApoA-I. Genomics. 2002;79(5):693-702.

30. Marbaix AY, Tyteca D, Niehaus TD, Hanson AD, Linster CL, Van Schaftingen E. Occurrence and subcellular distribution of the NADPHX repair system in mammals. Biochem J. 2014;460(1):49-58.

31. Clark JC, et al. Targeted disruption of the surfactant protein B gene disrupts surfactant homeostasis, causing respiratory failure in newborn mice. Proc Natl Acad Sci USA. 1995;92(17):7794-7798

32. Baranova I, et al. Lipopolysaccharide down regulates both scavenger receptor B1 and ATP binding cassette transporter A1 in RAW cells. Infect Immun. 2002;70(6):2995-3003.

33. Yin K, Liao DF, Tang CK. ATP-binding membrane cassette transporter A1 (ABCA1): a possible link between inflammation and reverse cholesterol transport. Mol Med. 2010;16(9-10):438-449.

34. Marbaix AY, Noël G, Detroux AM, Vertommen D, Van Schaftingen E, Linster CL. Extremely conserved ATP-or ADP-dependent enzymatic system for nicotinamide nucleotide repair. J Biol Chem. 2011;286(48):41246-41252.

35. Shumilin IA, et al. Identification of unknown protein function using metabolite cocktail screening. Structure. 2012;20(10):1715-1725.

36. Jha KN, et al. Biochemical and structural characterization of apolipoprotein A-I binding protein, a novel phosphoprotein with a potential role in sperm capacitation. Endocrinology. 2008;149(5):2108-2120.

37. Rudolph C, et al. ApoA-I-binding protein (AI-BP) and its homologues hYjeF_N2 and hYjeF_N3 comprise the YjeF_N domain protein family in humans with a role in spermiogenesis and oogenesis. Horm Metab Res. 2007;39(5):322-335.

38. Zhang M, et al. Apolipoprotein A-1 Binding Protein Inhibits Inflammatory Signaling Pathways by Binding to Apolipoprotein A-1 in THP-1 Macrophages. Circ J. 2018;82(5):1396-1404.

39. Zhang M, et al. AIBP reduces atherosclerosis by promoting reverse cholesterol transport and ameliorating inflammation in 
apoE-/- mice. Atherosclerosis. 2018;273:122-130.

40. Parsons PE, et al. Lower tidal volume ventilation and plasma cytokine markers of inflammation in patients with acute lung injury. Crit Care Med. 2005;33(1):1-6.

41. Bouros D, et al. The clinical significance of serum and bronchoalveolar lavage inflammatory cytokines in patients at risk for Acute Respiratory Distress Syndrome. BMC Pulm Med. 2004;4:6.

42. Meduri GU, et al. Persistent elevation of inflammatory cytokines predicts a poor outcome in ARDS. Plasma IL-1 beta and IL-6 levels are consistent and efficient predictors of outcome over time. Chest. 1995;107(4):1062-1073.

43. Tarling EJ. Expanding roles of ABCG1 and sterol transport. Curr Opin Lipidol. 2013;24(2):138-146.

44. Sweet DG, et al. European consensus guidelines on the management of neonatal respiratory distress syndrome in preterm infants--2013 update. Neonatology. 2013;103(4):353-368.

45. Spragg RG, et al. Effect of recombinant surfactant protein C-based surfactant on the acute respiratory distress syndrome. $N$ Engl J Med. 2004;351(9):884-892.

46. Meng H, et al. Exogenous surfactant may improve oxygenation but not mortality in adult patients with acute lung injury/acute respiratory distress syndrome: a meta-analysis of 9 clinical trials. J Cardiothorac Vasc Anesth. 2012;26(5):849-856

47. Dushianthan A, Cusack R, Goss V, Postle AD, Grocott MP. Clinical review: Exogenous surfactant therapy for acute lung injury/acute respiratory distress syndrome--where do we go from here? Crit Care. 2012;16(6):238.

48. Cho JY, et al. Inhibition of airway remodeling in IL-5-deficient mice. J Clin Invest. 2004;113(4):551-560.

49. Choi SH, Wiesner P, Almazan F, Kim J, Miller YI. Spleen tyrosine kinase regulates AP-1 dependent transcriptional response to minimally oxidized LDL. PLoS One. 2012;7(2):e32378.

50. Martínez-Sernández V, Orbegozo-Medina RA, Romarís F, Paniagua E, Ubeira FM. Usefulness of ELISA Methods for Assessing LPS Interactions with Proteins and Peptides. PLoS One. 2016;11(6):e0156530.

51. Kordonowy LL, et al. Obesity is associated with neutrophil dysfunction and attenuation of murine acute lung injury. Am $J$ Respir Cell Mol Biol. 2012;47(1):120-127.

52. Rudmann DG, et al. Modulation of allergic inflammation in mice deficient in TNF receptors. Am J Physiol Lung Cell Mol Physiol. 2000;279(6):L1047-L1057. 